\title{
Validation of Laser-Induced Fluorescent Photogrammetric Targets on Membrane Structures
}

\author{
Thomas W. Jones* \\ NASA Langley Research Center, Hampton, Virginia 23681 \\ Adrian A. Dorrington ${ }^{\dagger}$ \\ NASA Langley Research Center, Hampton, Virginia 23681 \\ Mark R. Shortis * \\ RMIT University, Melbourne, Australia \\ and \\ Aron R. Hendricks \\ James Madison University, Harrisonburg, Virginia
}

\begin{abstract}
The need for static and dynamic characterization of a new generation of inflatable space structures requires the advancement of classical metrology techniques. A new photogrammetric-based method for non-contact ranging and surface profiling has been developed at NASA Langley Research Center (LaRC) to support modal analyses and structural validation of this class of space structures. This full field measurement method, known as Laser-Induced Fluorescence (LIF) photogrammetry, has previously yielded promising experimental results. However, data indicating the achievable measurement precision had not been published. This paper provides experimental results that indicate the LIF-photogrammetry measurement precision for three different target types used on a reflective membrane structure. The target types were: (1) non-contact targets generated using LIF, (2) surface attached retro-reflective targets, and (3) surface attached diffuse targets. Results from both static and dynamic investigations are included.
\end{abstract}

\section{Introduction}

$\mathrm{P}$ hotogrammetry is an optical based measurement technique that can be applied to either still frame or video sequences, that derives three-dimensional (3D) surface data from recorded images, acquired at multiple perspectives, by triangulating the correspondence of intersecting rays for a point on an object ${ }^{1}$. A key requirement for photogrammetric measurement is the presence of high contrast targets on the object. When imaged using a camera, these targets must be suitable for sub-pixel centroid determination in the camera image plane. Laserinduced fluorescence has been successfully demonstrated as a non-contact technique capable of providing these high contrast targets ${ }^{2}$. While retro-reflective targets have been extensively studied and their performance well documented for photogrammetric applications ${ }^{3}$, laser-induced fluorescence (LIF) targets are a new concept for this area of metrology. A new generation of ultra-lightweight and inflatable "Gossamer" space structures have been defined as part of NASA's New Millennium Program. ${ }^{4}$ The proposed structures will be designed with materials that are very smooth, thin, and highly transparent and/or reflective, such as the LaRCTM_CP1 transparent polymer membranes developed at NASA Langley Research Center. Classic measurement techniques and tools such as accelerometers, strain gages, laser ranging and scanning devices and even classic photogrammetry find limited application in this new generation of space structures because of the material properties.

\footnotetext{
${ }^{*}$ Research Engineer, 4 Langley Blvd. M/S 236; non-Member.

${ }^{\dagger}$ National Research Council Associate, 4 Langley Blvd. M/S 236; Member

* Research Scientist, GPO Box 2476V; non-Member

${ }^{\S}$ Engineer, non-Member.
} 
The enabling mechanism for its use with these polymers is small concentrations of fluorescent dye that have been added to LaRC ${ }^{\text {TM}}$-CP1 polymers during their manufacture. Upon excitation with a laser source, the laser dye absorbs some of the incident laser energy, which is subsequently emitted via fluorescence at a slightly longer wavelength. This fluorescence is emitted in all directions thereby providing a predictable and repeatable dot pattern that can be viewed from any angle, and enabling the capture of high contrast images from the multiple viewing locations required for photogrammetric measurements. A typical example of the high contrast LIF targets is shown in Fig. 1.

Although LIF target generation has been successfully demonstrated for both static and dynamic measurements of membrane structures, ${ }^{5,6}$ no detailed study of measurement precision has yet been performed. This paper investigates and compares the photogrammetric measurement precision obtained using LIF targets with the measurement precision obtained using traditional retro-reflective and diffuse targets adhered to the object surface.

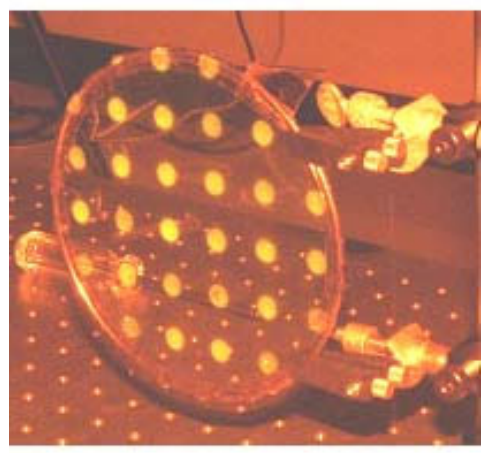

Figure 1: Laser Induced Fluorescence targets produced on transparent membrane.

\section{Test Setup}

A 1-meter square reflective coated polymer membrane was used as the test specimen for investigating the photogrammetric measurement precision achievable using LIF targets, surface attached retro-reflective targets, and surface attached diffuse targets. The test article is shown in Fig. 2. The membrane was tautly supported at the four corners and held within a rigid metal frame. The membrane average thickness was 25 microns. The membrane, while not representative of any particular space structure, does represent a candidate membrane material (CP1) that has a highly reflective coating on one side.

A 7-x 7 square grid of attached targets was used to define the polymer surface for the photogrammetric solution. A similar array of targets was created using laser-induced fluorescence. The fluorescent targets were generated by excitation with a pulsed, frequency-doubled Nd:YAG laser beam which was passed though a diffractive beam splitter. The LIF targets were laterally offset from the attached targets by approximately 1-inch. 


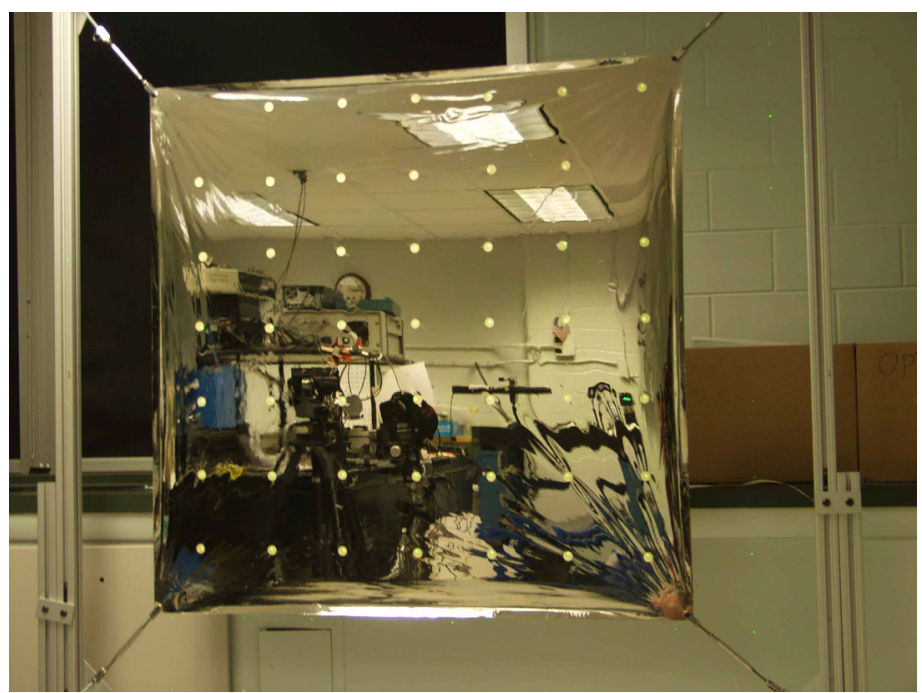

Figure 2: Test configuration - supported 1-meter reflective coated polymer membrane.

Images for the photogrammetric measurements were recorded synchronously by three 648-x 484-pixel (width-x height) progressive scan monochrome video cameras. The cameras were operated in asynchronous reset mode. The three cameras were arranged with two requirements in mind: (1) to provide the largest possible convergence angle between each camera pair while maintaining the reflective response from the retro-targets, and (2) to prevent "glint" reflections caused by the lighting required for the attached retro-reflective and diffuse targets. The graphic in Fig. 3 was produced as part of the analysis and provides a spatial model for the relative positions of the cameras and the 1meter square membrane. A coordinate system was established with the membrane being considered as roughly the $\mathrm{XZ}$ plane and the $+\mathrm{Y}$ axis coming out of the membrane towards the cameras. The cameras were located approximately 2 meters from the membrane. For both the static and dynamic tests, recorded images were analyzed using photogrammetric software tools to compute the 3D position for each target. Each camera was post-test calibrated to determine the principal point location, principal distance, and lens distortions. All images were digitized at 8-bit pixel depth. The imaged targets ranged in size from 6-12 pixels and had a gray scale intensity range of $45-255$.

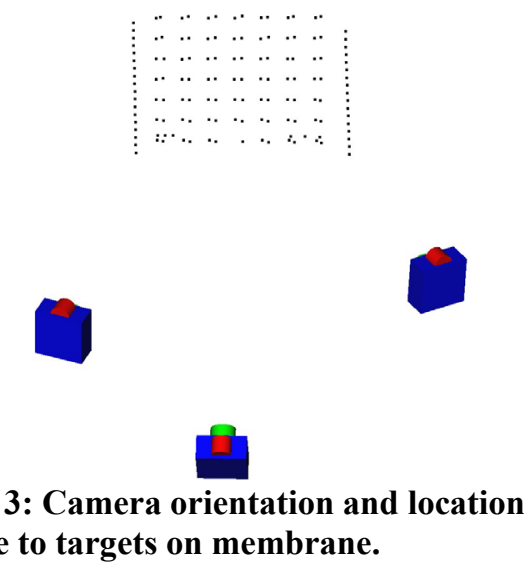

Figure 3: Camera orientation and location relative to targets on membrane. 
The range of intensity and contrast levels for the retro and diffuse targets is indicative of the type of problems encountered when using these types of targets on a reflective coated polymer. The LIF target intensity is low because this particular membrane was inadvertently manufactured with the laser dye concentration levels considerably lower than optimal. This problem could not be easily corrected because this membrane is the only one of its size and type yet to be manufactured. The dye concentration problem will be corrected when membranes are manufactured in the future. The additional lighting required to support the retro-reflective and diffuse targets also contributed to the degraded performance of the LIF targets. In several regions of the test article the gray scale separation from the target peak intensity to the adjacent background was only a gray scale value of 30 . However, this can be considered beneficial for the purposes of this investigation as the measurements presented here were performed under worst-case conditions. The image in Fig. 4 shows an actual image extracted from the recorded sequence. The inset window highlights the typical contrast between the targets and the background for images in the recorded sequence. The average greyscale intensity values for the LIF, diffuse, and retro-reflective targets shown in the Fig. 4 inset were 35, 151, and 255, respectively.

\section{Target Profiles}

Prior research has shown that target centroid detection algorithms will produce the most accurate results when the target intensity profiles are Gaussian in shape [reference 5].

The current experiment produced a varied range of target profiles for the target types being compared. The examples shown in Fig. 5, 6, \& 7 were all selected from the first image in the recorded sequence and show the range and extremes of target quality present in the scene. Intensity profiles for the retro-reflective targets, widely considered by the metrology community to be the best possible targets for photogrammetry use, are shown in Fig. 5. The intensity profiles shown in Fig. 5 range from suitably exposed and Gaussian-like shaped to significantly over exposed and "flat-topped". Previous studies have shown the affect of target saturation on overall centroiding precision to be typically 0.001 inches, but somewhat dependent on the processing algorithm ${ }^{7}$. Intensity profiles for the diffuse yellow targets are shown in Fig. 6. These profiles have a slightly worse profile than the retro-reflective targets, but noticeably better than the intensity profiles for the LIF targets shown in Fig. 7.

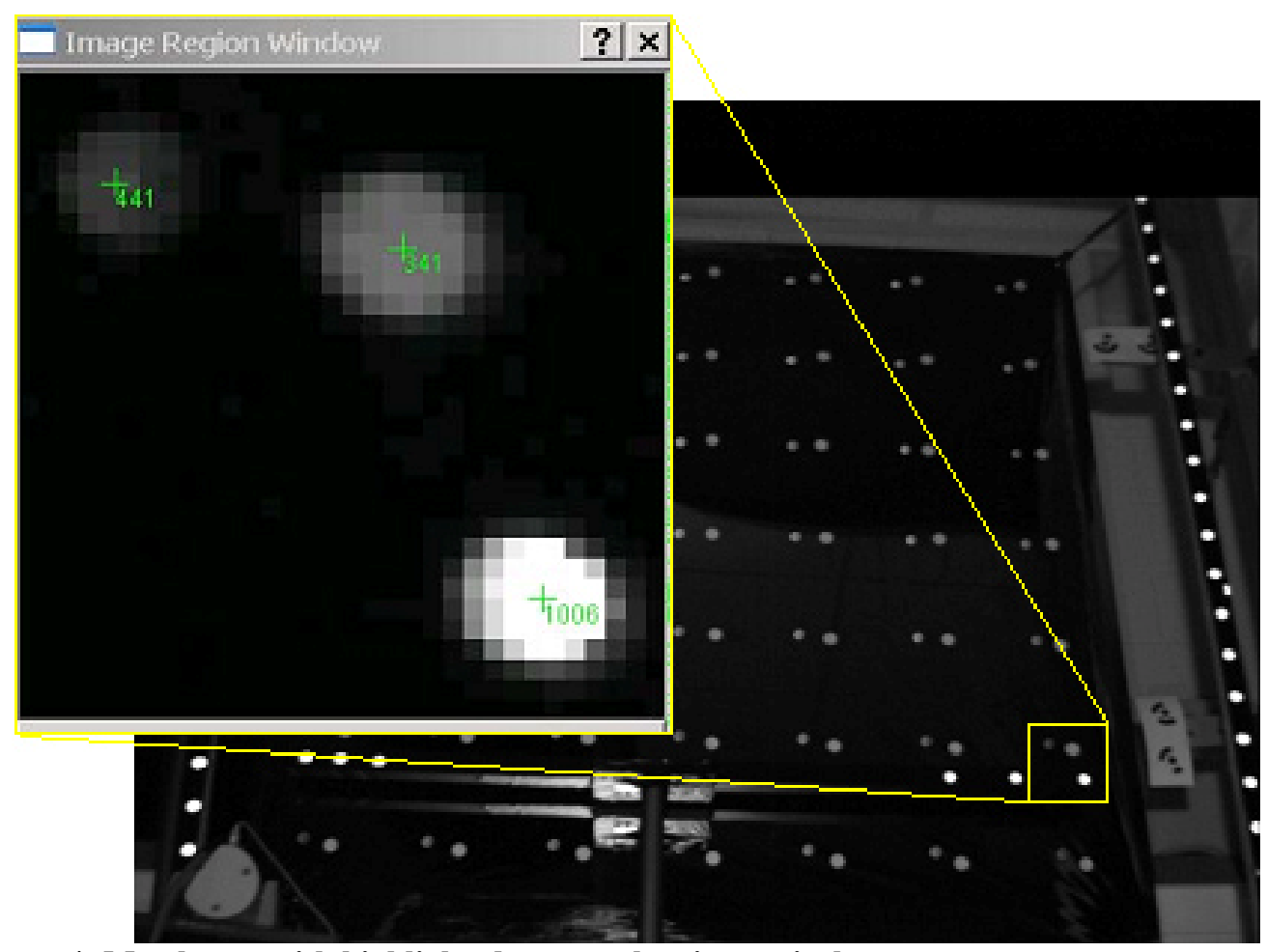

Figure 4: Membrane with highlighted targets showing typical target contrast. 


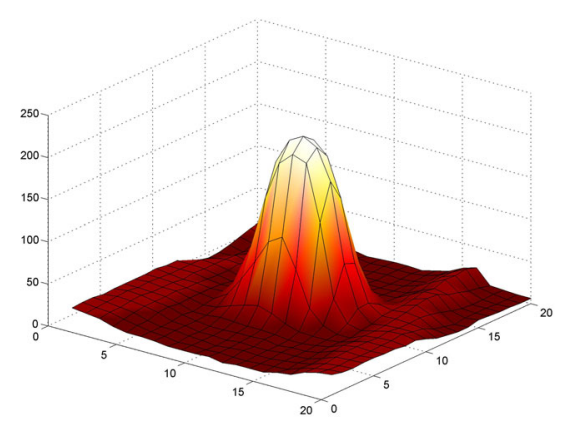

Figure 5: a) Retro-reflective Target (Good)

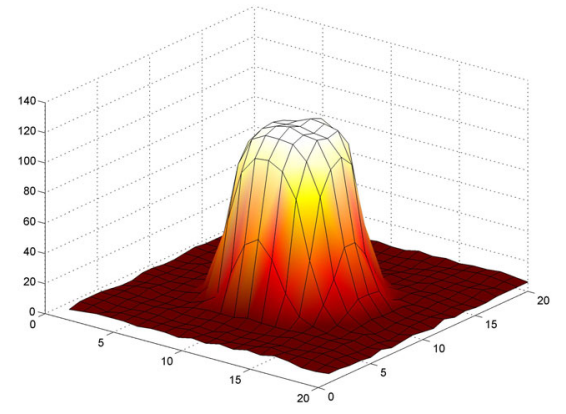

Figure 6: a) Diffuse Yellow Target (Good)

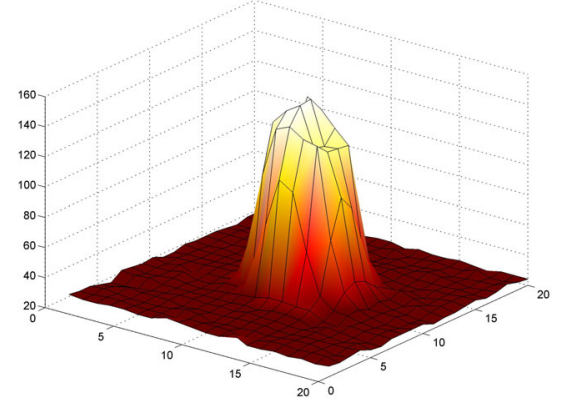

Figure 7: a) Laser Induced Target (Good)

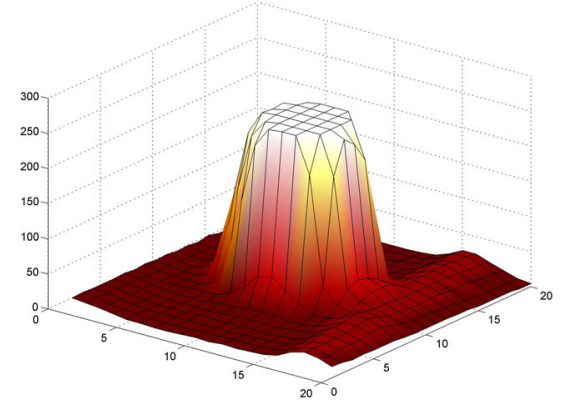

b) Retro-reflective Target (Poor)

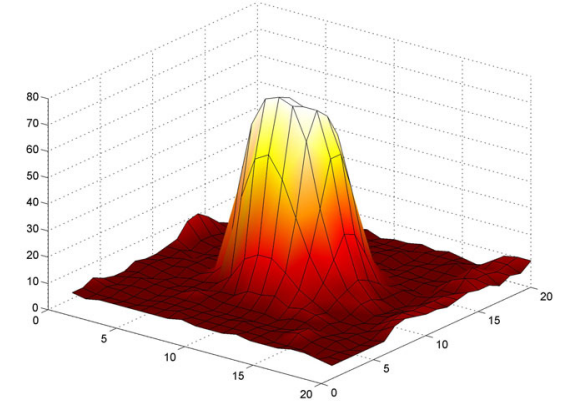

b) Diffuse Yellow Target (Poor)

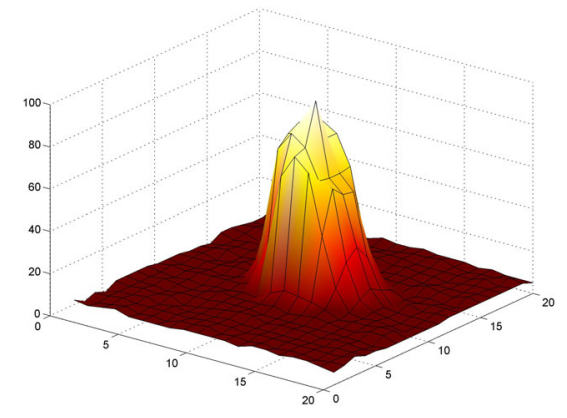

b) Laser Induced Target (Poor)

The major disadvantage associated with the diffuse targets is the flood-type light requirement and the generally long exposure times needed to obtain sufficient contrast. Both of these requirements limit the effectiveness of diffuse targets for dynamic measurement. The LIF target intensity profiles shown in Fig. 7 range from slightly misshapen to very irregularly shaped, and in the worst case exhibiting a significantly brighter pixel in the center of the target. The main reason for these poor profiles is the use of a multi-spatial-mode laser for the dot pattern projection. This laser was the only suitable pulsed laser available at the time of the experiment and although the targets it generates are of relatively poor quality, it does represent the worst-case condition from which precisions can only improve. From the target profiles presented in Fig. 5-7, it is reasonable to predict that the retro-reflective targets will have the highest precision, followed closely by the yellow stick-on targets, and the LIF targets will have the lowest.

For comparison, the fluorescent target profile was experimentally obtained using a single mode continuous-wave Nd:YAG laser (Fig. 8), which shows an almost ideal profile that is arguably better than the retro-reflective targets. The Gaussian profile of the laser beam reproduced a corresponding profile in the fluorescent target that is ideally suited to least-squares Gaussian centroiding, and should provide very high precision results. Although this particular laser is not suitable for dynamic measurement, single mode pulsed lasers are very common and a similar pulse profile could have been obtained for dynamic measurements 


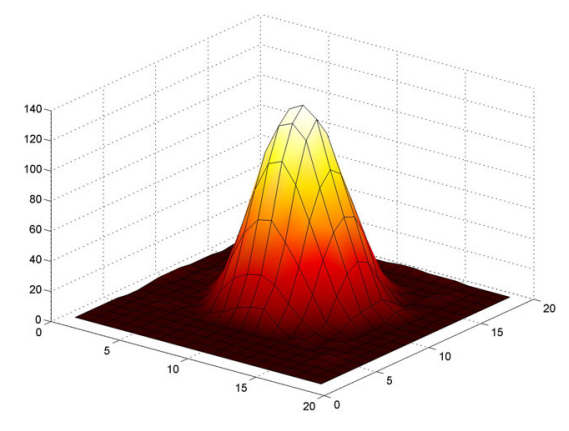

Figure 8: Example of single mode laser-induced fluorescence target profile.

\section{Static Test}

Static tests were conducted to identify the baseline uncertainty associated with each of the three targeting techniques. Testing in a static manner eliminated potential error sources such as (a) camera synchronization errors, (b) errors caused by target motion during the camera exposure, and (c) changes in target illumination resulting from object motion. For the static testing, the lighting and laser illumination were adjusted so all three target types were visible to the cameras. This enabled simultaneous imaging of the three target types, in addition to the targets on the membrane, retro-reflective targets were attached to the rigid frame supporting the membrane. The targets on the rigid frame were used to assess membrane stability and provide a best-case reference for comparing measurement precision. Extreme care was taken to minimize all air currents during the experiment that could result in undesired motion of the membrane. Results obtained from the static tests were later used as a baseline reference for comparison of results obtained during dynamic testing.

\section{Processing}

Processing photogrammetric images to obtain accurate 3D spatial target coordinates from 2D imagery requires extremely precise target centroid detection in the image plane. For the current experimental setup, a target centroid detection error of 0.01 pixel is estimated to yield a target spatial coordinate error of $0.001 \mathrm{inch}$. Many factors play into the overall target centroid determination including target intensity, size, and quantization level. Background lighting can also be a significant source of error as it impacts the ability of processing algorithms to accurately detect target boundaries by thresholding. Consistent comparison of the results from the three target types used in this experiment required that each target type be visible and simultaneously recorded by each camera. This requirement created a need for additional lighting that would otherwise be undesirable if using only LIF targets. The expected net effect is a loss in precision for the LIF targets due to the reduction in target contrast.

The numerical precision of the centroid detection processing algorithm was investigated by copying a single image 500 times to simulate a recorded sequence with absolutely with no target motion. The results indicated the errors in spatial coordinate determination attributable to processing algorithm numerical uncertainty were less than 0.001 inches.

\section{Analysis}

Several centroiding algorithms have been developed for processing photogrammetric data and are now the cornerstone of many commercially available photogrammetric software packages. In many cases the software provides the user with the option to choose which centroiding algorithm is used for processing. Two different, commercially available photogrametry analysis software packages were used in the present study to ensure unbiased results. Both packages offered the choice of multiple techniques for centroid computation. Three of the more common centroid detection techniques were chosen for study - two variations on a weighted centroid method, and a Gaussian least squares fit method. The basic algorithms are intensity based techniques and have been previously studied as part of a broader evaluation for target centroiding precision ${ }^{8}$. 
A baseline precision was established by examining the standard deviation of each target group (i.e. retro, diffuse, and LIF) over a 500-frame sequence while the membrane was stationary. The graphs in Fig. 13 represent the $3 \sigma$ ( $99 \%$ confidence level) standard deviation for each target group averaged for three separately recorded data sets. The data are presented as a measure of the scatter sX, sY, and sZ, in three-dimensional coordinate space $\mathrm{x}, \mathrm{y}, \mathrm{z}$, where the y spatial coordinate is the out-of-plane direction relative to the membrane. The error bars indicate plus and minus one standard deviation of the results from these three runs.

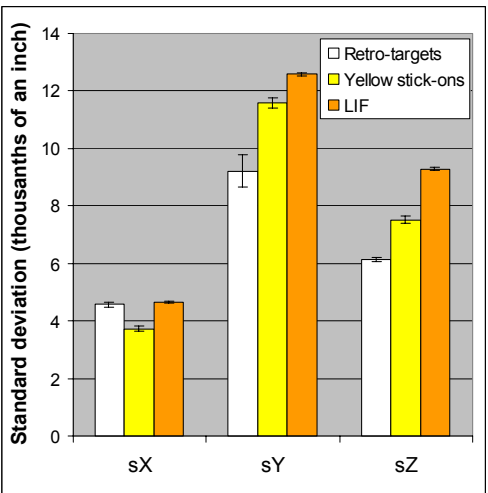

a) Centroid Method

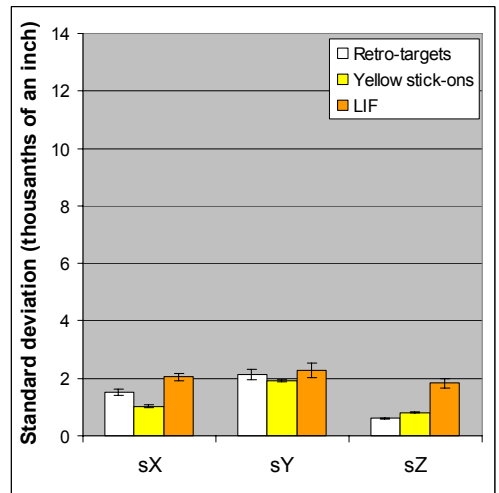

b) Gaussian Least Squares Method

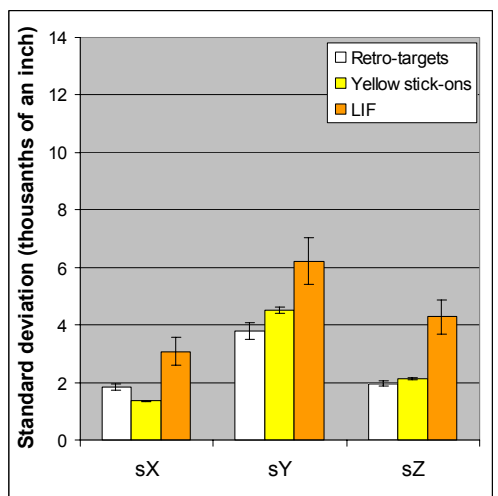

c) Weighted Centroid Method

Figure 9: Computed standard deviations for static measurements using three different processing algorithms

The results obtained by processing the imagery using the centroid methods, Fig. 9 a) and c) show the expected trend from examination of the spot profiles, with the retro-reflective targets having the smallest scatter followed closely by the diffuse attached targets and the LIF having the highest. The results produced using the Gaussian least squares method show significantly less dependence on target spot profile quality. In some cases, results for the retro-reflective targets are slightly worse than the diffuse yellow targets. This is probably because there were a higher proportion of retro-reflective targets with a saturated intensity profile compared to the yellow diffuse targets, resulting in a biased average value. In addition, with the retro-reflective targets being rigidly mounted the possible effects of air currents moving the polymer and the attached and fluorescence targets can be discounted. In all cases, the out-of-plane (y spatial coordinate) dimension exhibited slightly worse precision than the in-plane dimensions, which is typical of photogrammetric solutions when the cameras are not at the optimum locations for intersection angles of 90 degrees at the targets.

\section{Dynamic Test}

Structural dynamic characterization is a key requirement in supporting the validation of the new class of membrane structures. LIF targeting eliminates many of the problems associated with traditional adhesive retroreflective or diffuse targets, such as: (1) added structural mass that will affect the dynamics of the structure, (2) the laborious process of adhering adhesive targets to the structure, and (3) the inability to remove adhesive targets without damaging the membrane material. LIF targeting also provides enhanced capabilities for dynamic measurements since a pulsed laser (10 ns typical pulse duration) can be used to obtain simultaneous, instantaneous measurements of all illuminated target locations. This is in stark contrast to visible light dot projection, which cannot be used for dynamic measurements because of the long camera exposure times required for the cameras to collect enough diffuse scattered light to resolve the targets ${ }^{7}$.

The same experimental setup shown in Fig. 2 was used for dynamic testing. However, the membrane was forced into a steady, periodic oscillation using a short-stroke electrodynamic shaker attached to one corner. The shaker provided a $2.3 \mathrm{~Hz}$ sinusoidal excitation input to the membrane, as previous measurements indicated this structure had a very predominant vibration mode at this frequency ${ }^{2}$. Additionally, a scanning laser vibrometer was used as a high precision standard for qualitative validation of the photogrammetry results. The vibrometer was configured to sequentially measure the same 7-x 7 grid of attached targets used for the photogrammetric solution. Note however, that the vibrometer could not measure all target locations simultaneously, whereas the photogrammetry system 
could. Unfortunately each system lacked a common phase reference that prevented a quantitative comparison and reconstructing an oscillation by assembling data collected over multiple runs.

The photogrammetric measurements of target displacement were derived from recorded video data containing a sequence of 500 frames. The pulsed, frequency-doubled Nd:YAG laser was synchronized with the video cameras to provide one 10-ns pulse of illumination per video frame, and the exposure time of the cameras was set to $1 / 8000$ second. The video data was processed using photogrammetric algorithms to obtain the out-of-plane target displacement data. Structural phase and amplitude information was derived from the displacement data by performing a FFT of the time-dependent displacement measured for each target. For comparison, the target displacements were also obtained by integrating the target velocity measurements acquired using the scanning laser vibrometer. Normalized mode shapes measured using the laser vibrometer, LIF targets, and surface attached targets are shown in Fig. 10. The results shown in Fig. 10 show good qualitative agreement between the measurement techniques. While the intent of this portion of the study was to provide data for quantitative comparison, flaws in the experimental setup for dynamic recording only allowed for qualitative comparison.

While the vibrometer is capable of high quality measurements at high sample rates, it can only measure one point at time. This form of data acquisition is slow and can take many hours to complete a minimum survey of a test article like this membrane. Videogrammetry is an attractive alternative, especially when combined with LIF targeting, because of its ability to measure all points simultaneously.
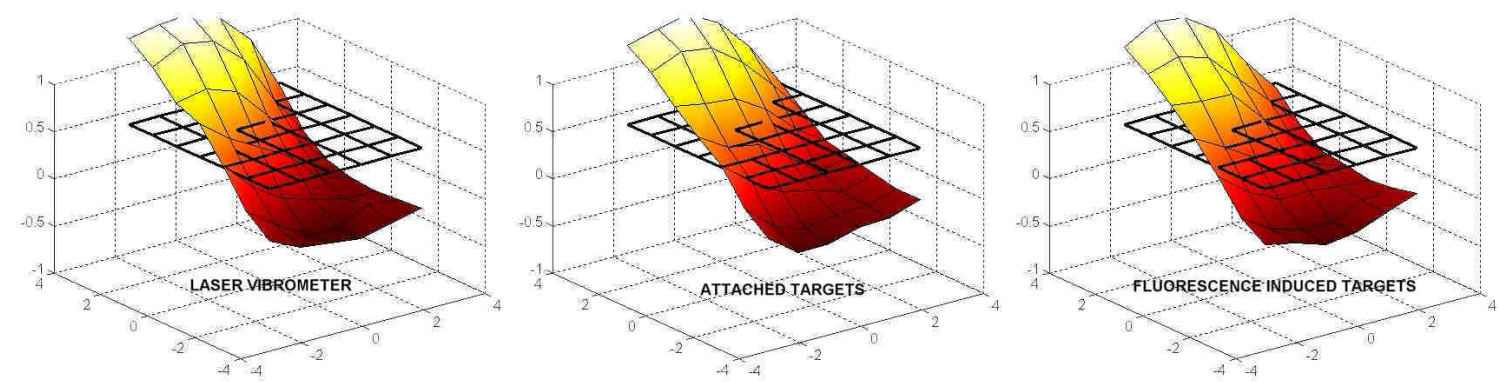

Figure 10: Dynamic Response

Common modal points for each of three measurement techniques: laser vibrometer, attached targets, and LIF targets.

\section{Conclusion}

The experimental data presented in this paper indicates that laser-induced fluorescence (LIF) target generation techniques are a viable alternative to physically attached targets for static and dynamic characterization of membrane structures. While many factors contribute to the best obtainable precision in a photogrammetric measurement project, most notably camera calibration, camera convergence, targeting coverage and subpixel centroid determination are the critical issues. The focus of this study was the effects on centroid determination. The LIF target properties were not significantly worse than those of surface-attached retro-reflective targets, commonly regarded within the metrology community as the best possible targets for photogrammetric measurements. Correspondingly, it has been shown that the determination of LIF target centroid locations can be performed at a level of precision comparable to surface-attached retro-reflective and diffuse targets. Relative differences in precision between the retro-reflective targets and the LIF targets in the static test was typically less than 0.002 " in either the $\mathrm{x}, \mathrm{y}$, or $\mathrm{z}$ planes for a given processing algorithm. The major difference in absolute precisions for different algorithms was attributed to the processing characteristics of these algorithms.

Examination of the various target intensity profiles showed that LIF targets can be produced with an almost ideal Gaussian distribution, but as also showed less desirable profiles are possible if appropriate attention is not given to laser selection and optical alignment.

While dynamic characterization has previously been the exclusive domain of attached targets, it has been shown that LIF target generation is capable of providing the necessary contrast for the short exposures needed to support the sampling frequencies typical of this type of analysis. The experiment identified modal frequencies through data acquired with LIF photogrammetry that was shown to be consistent with data acquired with more traditional attached target photogrammetry and high precision laser vibrometry. 
The comparable precision of LIF target generation, coupled with its unique advantages over attached targets, gives metrology developers an alternative in applications requiring discrete targets for surface shape measurement. The benefits of LIF target generation are significant when considering the potential negative effects on the structural properties of membranes when using attached targets. For this experiment, LIF target generation in worst-case conditions has provided only slightly degraded precision to that obtained with more traditional techniques.

\footnotetext{
${ }^{1}$ Atkinson K. B. (editor), "Close range photogrammetry and Machine Vision”, Whittles Publishing, Scotland, UK, 2001, pp25-26.

${ }^{2}$ Dorrington A.A., Jones T.W., Danehy P.M., "Laser-induced fluorescence photogrammetry for profiling gossamer space structures," Conference on Lasers and Electro-optics 2003, Baltimore, Maryland, June 2003.

${ }^{3}$ Clarke, T.A., "Analysis of the properties of targets used in digital close-range photogrammetric measurement," Proceedings of SPIE, Videometrics III, Boston, Mass., pg 251-263.

${ }^{4}$ Jenkins, C.H.M. (editor), Gossamer Spacecraft: Membrane and Inflatable Structures Technology for Space Applications, Vol. 191, Progress in Astronautics and Aeronautics, AIAA, Reston, VA, 2001.

${ }^{5}$ Dorrington A.A., Jones T.W., Danehy P.M., "Laser-induced fluorescence photogrammetry for dynamic characterization of transparent and aluminized membrane structures," 39rd AIAA/ASME/SAE/ASEE Joint Propulsion Conference, AIAA Paper 2003-4798, July 2003.

${ }^{6}$ Jones, T.W., Dorrington A.A., Brittman P.L., Danhey P.M., "Laser induced fluorescence for photogrammetric measurement of transparent or reflective aerospace structures," Proceedings of the 49th International Instrumentation Symposium, Orlando, Florida, 2003.

${ }^{7}$ Shortis, M.R., Clarke, T.A., and Short, T., "A comparison of some techniques for the subpixel location of discrete target images," Proceedings of SPIE, Videometrics III, Boston, Mass., pg 239-250; 1994.

${ }^{8}$ Shortis, M.R., Clarke, T.A., Robson, S., "Practical testing of the precision and accuracy of target image centering algorithms," Proceedings of SPIE, Videometrics IV, Vol. 2598, pg 65-76; 1995.
} 